Check for updates

Cite this: RSC Adv., 2018, 8, 22452

\title{
First-principles study on the electrical and thermal properties of the semiconducting $\mathrm{Sc}_{3}(\mathrm{CN}) \mathrm{F}_{2}$ MXene†
}

\author{
Kan Luo, (D) ab Xian-Hu Zha, ${ }^{\mathrm{b}}$ Yuhong Zhou, ${ }^{\mathrm{b}}$ Zhansheng Guo, (D) Cheng-Te Lin, ${ }^{\mathrm{d}}$ \\ Qing Huang, (iD ${ }^{b}$ Shenghu Zhou, (D) *a Ruifeng Zhang (D) ${ }^{\text {e }}$ and Shiyu Du (D) *b
}

\begin{abstract}
The two-dimensional materials MXenes have recently attracted interest for their excellent performance from diverse perspectives indicated by experiments and theoretical calculations. For the application of MXenes in electronic devices, the exploration of semiconducting MXenes arouses particular interest. In this work, despite the metallic properties of $\mathrm{Sc}_{3} \mathrm{C}_{2} \mathrm{~F}_{2}$ and $\mathrm{Sc}_{3} \mathrm{~N}_{2} \mathrm{~F}_{2}$, we find that $\mathrm{Sc}_{3}(C N) \mathrm{F}_{2}$ is a semiconductor with an indirect band gap of $1.18 \mathrm{eV}$, which is an expansion of the semiconducting family members of MXene. Using first-principles calculations, the electrical and thermal properties of the semiconducting $\mathrm{SC}_{3}(\mathrm{CN}) \mathrm{F}_{2} \mathrm{MXene}$ are studied. The electron mobilities are determined to possess strong anisotropy, while the hole mobilities show isotropy, i.e. $1.348 \times 10^{3} \mathrm{~cm}^{2} \mathrm{~V}^{-1} \mathrm{~s}^{-1}$ along $x, 0.319 \times$ $10^{3} \mathrm{~cm}^{2} \mathrm{~V}^{-1} \mathrm{~s}^{-1}$ along the $y$ directions for electron mobilities, and $0.517 \times 10^{3} \mathrm{~cm}^{2} \mathrm{~V}^{-1} \mathrm{~s}^{-1}$ along $x, 0.540 \times 10^{3} \mathrm{~cm}^{2} \mathrm{~V}^{-1} \mathrm{~s}^{-1}$ along the $y$ directions for hole mobilities. The room-temperature thermal conductivity along the $\Gamma \rightarrow M$ direction is determined to be $123-283 \mathrm{~W} \mathrm{~m}^{-1} \mathrm{~K}^{-1}$ with a flake length of 1-100 $\mu \mathrm{m}$. Besides, $\mathrm{SC}_{3}(\mathrm{CN}) \mathrm{F}_{2}$ presents a relatively high specific heat of $547 \mathrm{~J} \mathrm{~kg}^{-1} \mathrm{~K}^{-1}$ and a low thermal expansion coefficient of $8.703 \times 10^{-6} \mathrm{~K}^{-1}$. Our findings suggest that the $\mathrm{Sc}_{3}(\mathrm{CN}) \mathrm{F}_{2} \mathrm{MXene}$ might be a candidate material in the design and application of 2D nanoelectronic devices.
\end{abstract}

Received 21st April 2018

Accepted 28th May 2018

DOI: $10.1039 / c 8 r a 03424 a$

rsc.li/rsc-advances solutions. ${ }^{8,9}$ The terminations on MXenes are typically functionalized by $-\mathrm{H},-\mathrm{F},=\mathrm{O}$, and $-\mathrm{OH}$ groups coming from $\mathrm{HF}$ or $\mathrm{H}_{2} \mathrm{O} \cdot{ }^{10-12}$ Naguib et al. ${ }^{1}$ have denoted the functionalized MXenes as $\mathrm{M}_{n+1} \mathrm{X}_{n} \mathrm{~T}_{x}$, with $\mathrm{T}$ standing for the surface-terminating group. Recently, about 20 different MXenes have been reported, ${ }^{13}$ and the family of MXenes has been expanded to double transition metals carbides $\mathrm{M}_{2}^{\prime} \mathrm{M}^{\prime \prime} \mathrm{C}_{2}$ and $\mathrm{M}_{2}^{\prime} \mathrm{M}^{\prime \prime}{ }_{2} \mathrm{C}_{3}{ }^{14}$ The large number of theoretically possible members of the MXene family, the diversity of physical properties among MXenes, and their relative convenience in synthesis merit the attraction of these compounds for novel production methods and 2D materialrelated potential applications..$^{15}$ For example, Xu et al. reported the growth of high quality crystalline MXenes achieved by a chemical vapor deposition technique. ${ }^{16}$ Azofra et al. investigated the $\mathrm{N}_{2}$ capture and ammonia conversion behaviour of $\mathrm{d}^{2}-\mathrm{d}^{4}$ MXenes, ${ }^{17}$ and the $\mathrm{CO}_{2}$ capture and conversion may be another possible application of the MXene materials. ${ }^{18}$ Some of the MXenes are demonstrated to be topological insulators, ${ }^{19,20}$ exhibiting multiple Dirac cones and giant spin-orbit splitting. ${ }^{21}$ Ashton et al. compared the thermodynamic stability of 54 MXenes, ${ }^{22}$ finding Sc-based MXenes to be highly stable with $\mathrm{F}$ termination, and the low diffusion barriers for Li on fluorinated MXene surfaces ${ }^{23}$ can make $\mathrm{Sc}_{n+1} \mathrm{X}_{n} \mathrm{~F}_{2}$ MXenes possible candidates for electrode materials in Li-ion batteries. Many of the recent studies on MXenes have been focused on the electronic, magnetic, catalytic or thermoelectric properties. ${ }^{24-31}$ Liu et al. 
systematically explored the electronic properties of Sc-based MXenes by first-principles calculations. ${ }^{32}$ Wang et al. investigated the band gap tuning of $\mathrm{Sc}_{2} \mathrm{C}$ MXene for optoelectronic devices by changing the types of surface chemical groups, ${ }^{33}$ and heterostructures based on three different functionalized $\mathrm{Sc}_{2} \mathrm{C}$ MXenes were built to investigate the possible application for nanodevices. ${ }^{34}$ The data from these works suggest that MXenes are promising as electronic devices, for which the semiconducting members are generally desired. However, most MXenes are metallic due to the inheritance of the conducting feature of the electronic band structures in transition metal carbides or nitrides. Therefore, further investigation of these materials is needed, such as the effect of compositional modification on electronic properties as well as structural stability, in order to expand the MXene family, especially for intrinsically semiconducting ones. ${ }^{35}$

In this work, the band structures of three fluorinefunctionalized scandium MXenes $\mathrm{Sc}_{3} \mathrm{C}_{2} \mathrm{~F}_{2}, \quad \mathrm{Sc}_{3} \mathrm{~N}_{2} \mathrm{~F}_{2}$ and $\mathrm{Sc}_{3}(\mathrm{CN}) \mathrm{F}_{2}$ are studied using density functional theory (DFT). Here, we demonstrate that $\mathrm{Sc}_{3}(\mathrm{CN}) \mathrm{F}_{2}$ is a semiconductor with an indirect band gap of $1.18 \mathrm{eV}$ from the Heyd-Scuseria-Ernzerhof (HSE06) correction. This demonstrates that the design of new semiconducting MXenes is possible. The electronic, carrier mobility and thermal properties of the $\mathrm{Sc}_{3}(\mathrm{CN}) \mathrm{F}_{2}$ MXene are also predicted via theoretical calculations. The strong anisotropy in electron mobility has been determined. In addition, the relatively high specific heat and low thermal expansion coefficient make $\mathrm{Sc}_{3}(\mathrm{CN}) \mathrm{F}_{2}$ a good candidate material for nanoelectronic devices.

\section{Computational details}

The first-principles calculations are carried out based on projector augmented-wave (PAW) potentials ${ }^{36}$ in reciprocal space represented by a generalized gradient approximation $(\mathrm{GGA})^{37}$ in density functional theory with Perdew-BurkeErnzerhof (PBE) for the exchange-correlation function as implemented in the VASP codes..$^{38}$ Plane-waves with energies up to $550 \mathrm{eV}$ are employed to describe the electronic wave functions, in which the Sc $3 \mathrm{p}^{6} 3 \mathrm{~d}^{1} 4 \mathrm{~s}^{2}, \mathrm{C} 2 \mathrm{~s}^{2} 2 \mathrm{p}^{2}, \mathrm{~N} 2 \mathrm{~s}^{2} 2 \mathrm{p}^{3}$ and $\mathrm{F} 2 \mathrm{~s}^{2} 2 \mathrm{p}^{5}$ electrons are considered as valence states. To avoid any artificial interaction between the layers and their images, a $30 \AA$ lattice parameter in the $c$-axis perpendicular to the MXene surface is set. In the optimized structures, the maximum force on each atom is less than $10^{-4} \mathrm{eV} \AA^{-1}$. The total energies are converged within $10^{-6} \mathrm{eV}$. For the structural optimization, the Brillouin zone (BZ) is sampled using a set of $\Gamma$-centered $12 \times 12 \times 1 \mathrm{k}$-points. Due to the underestimation of energy band gaps through GGA-PBE, ${ }^{39}$ the non-local HSE06 hybrid functional is also adopted to correct the band gap values. $^{32,40}$

The carrier mobilities of the $\mathrm{Sc}_{3}(\mathrm{CN}) \mathrm{F}_{2}$ MXene are calculated using the deformation potential (DP) theory ${ }^{41-43}$ based on an orthorhombic unit cell, as the yellow rectangle highlights in Fig. 1(a). The carrier mobility has been calculated according to eqn $(1)^{44,45}$

$$
\mu=\frac{e \hbar^{3} C}{k_{\mathrm{B}} T m^{*} m_{\mathrm{a}}\left(E^{i}\right)^{2}}
$$

where $\hbar$ and $k_{\mathrm{B}}$ are the reduced Planck and Boltzmann constants, respectively. $T$ denotes temperature, and $m^{*}$ is the carrier effective mass along the transport direction; $m_{\mathrm{a}}$ is calculated by $m_{\mathrm{a}}=\sqrt{m_{x}^{*} m_{y}^{*}}$, where $m_{x}^{*}$ and $m_{y}^{*}$ are the carrier effective masses along the $x$ and $y$ directions, respectively, as shown in Fig. 1(c). $C$ is the elastic modulus along the transport direction, determined by extrapolation based on the relationship of $C(\Delta a / a)^{2} / 2=\left(E-E_{0}\right) / S_{0}$, where $\left(E-E_{0}\right)$ is the change of the total energy under a small lattice variation $\Delta a$ from the equilibrium lattice constant $a_{0}$ along the transport direction, with a small step size $\left(\Delta a / a_{0} \sim 0.5 \%\right)$, and $S_{0}$ is the area of the lattice in the $x y$ plane. Finally, $E^{i}$ is the deformation potential constant of the valence band maximum (VBM) for holes or the conduction band minimum (CBM) for electrons along the transport direction, calculated by $E^{i}=\Delta V_{i} /\left(\Delta a / a_{0}\right)$ with $\Delta V_{i}$ as the energy change of the $i^{\text {th }}$ energy band. The deformation potential constant is estimated as the slope of the linear fitting. function between $\Delta V_{i}$ and $\Delta a / a_{0}$.

The thermal conductivities have been calculated from the phonon dispersion of a hexagonal unit cell, as the gray rhombus marks in Fig. 1(a). The phonon thermal conductivity was calculated within the framework of Klemens' theory ${ }^{46,47}$

$$
\kappa_{\mathrm{p}}=\frac{\rho}{T} \sum_{j} \frac{\left\langle v_{j}\right\rangle^{4}}{\left\langle\gamma_{j}^{2}\right\rangle \omega_{\max , j}} \ln \frac{\omega_{\max , j}}{\omega_{\min , j}}
$$

where $\rho$ is the mass density, calculated by $\rho=\frac{M}{\left(\frac{\sqrt{3}}{2} a^{2} d\right)}$, with $M$ being the mass of the MXene unit cell, $a$ is the lattice parameter in the $x y$ plane, and $d$ denoting the MXene layer

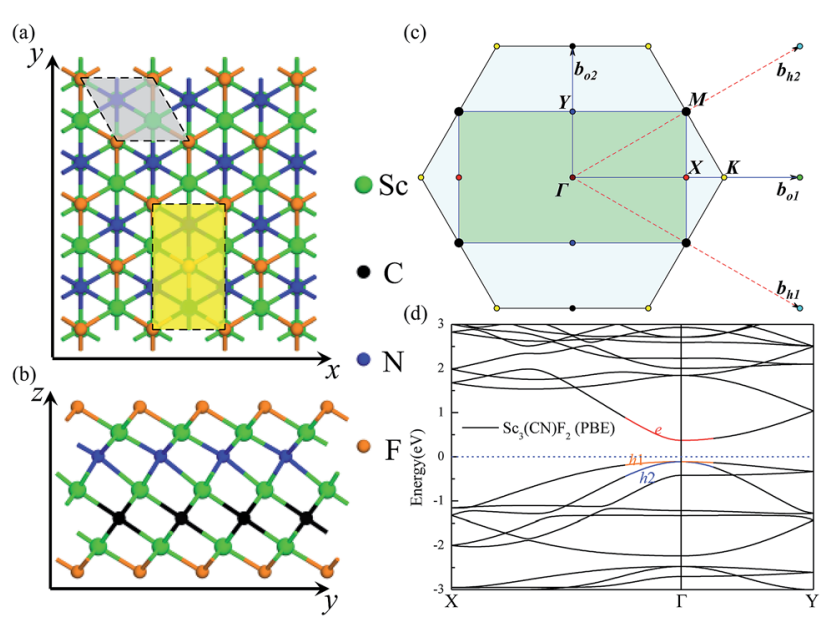

Fig. 1 The top view (a) and side view (b) of the $\mathrm{Sc}_{3}(\mathrm{CN}) \mathrm{F}_{2}$ MXene. (c) The Brillouin zone of the 2D hexagonal and orthorhombic lattice, the high symmetry routes $I \rightarrow K(I \rightarrow X)$ and $I \rightarrow M(I \rightarrow Y)$ correspond to the real-space $x$ and $y$ directions, respectively. (d) The band structure of the $\mathrm{Sc}_{3}(\mathrm{CN}) \mathrm{F}_{2}$ MXene based on the orthorhombic cell with the Fermi level located at zero. The atoms are represented by spheres: Sc (green), C (black), N (blue) and F (orange). 
thickness. ${ }^{48}$ A bilayer $\mathrm{Sc}_{3}(\mathrm{CN}) \mathrm{F}_{2}$ MXene structure model is optimized to calculate the layer thickness. The value of $d=$ 10.284 $\AA$ is measured as the distance between two middle layer Sc atom planes in the bilayer $\mathrm{Sc}_{3}(\mathrm{CN}) \mathrm{F}_{2}$ MXene. To accurately describe the interlayer interaction of the bilayers for $\mathrm{Sc}_{3}(\mathrm{CN}) \mathrm{F}_{2}$, a zero damping van der Waals (vdW) correction (DFT-D3) of Grimme ${ }^{49}$ has been adopted. $v_{j}, \omega_{\max , j}$ and $\omega_{\min , j}$ are the group velocity and the maximum and minimum circular frequency of each $j^{\text {th }}$ branch, respectively. Due to the finite flake length $L$, the term of $\omega_{\min , j}$ is redefined as $\omega_{\min , j}=\left(\frac{M\left\langle v_{j}\right\rangle^{3} \omega_{\max , j}}{2\left\langle\gamma_{j}{ }^{2}\right\rangle k_{\mathrm{B}} T L}\right)^{1 / 2}$, where $\gamma_{j}$ is the average value of the branch Grüneisen parameter, and $\left\langle\gamma_{j}^{2}\right\rangle$ in eqn (2) is estimated by $\left\langle\gamma_{j}{ }^{2}\right\rangle=\frac{\sum_{k} \gamma_{j, k}^{2} c_{\mathrm{v}}(j, k)}{\sum_{k} c_{\mathrm{v}}(j, k)}$. Phonopy software ${ }^{50}$ combined with the VASP code is utilized for phonon

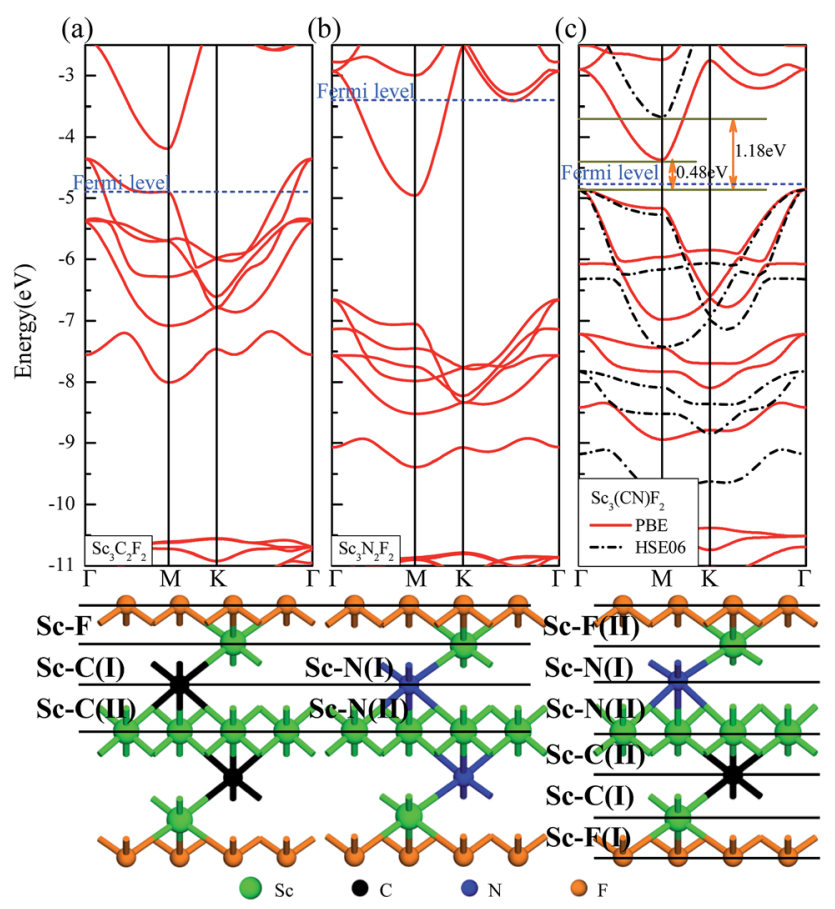

Fig. 2 Band structures of three $F$ terminated MXenes $\mathrm{SC}_{3} \mathrm{C}_{2} \mathrm{~F}_{2}$ (a), $\mathrm{Sc}_{3} \mathrm{~N}_{2} \mathrm{~F}_{2}$ (b) and $\mathrm{SC}_{3}(\mathrm{CN}) \mathrm{F}_{2}$ (c), and the vacuum energy is set as zero. Red solid and black dotted lines represent electronic energy bands from GGA-PBE and HSEO6 respectively. The side view of the $\mathrm{Sc}_{3} \mathrm{C}_{2} \mathrm{~F}_{2}$, $\mathrm{SC}_{3} \mathrm{~N}_{2} \mathrm{~F}_{2}$ and $\mathrm{SC}_{3}(\mathrm{CN}) \mathrm{F}_{2}$ MXenes are shown below each band structure figure respectively. dispersion calculations. The theoretical calculation is performed with density functional perturbation theory (DFPT), ${ }^{51}$ and a $6 \times 6 \times 1 \mathrm{k}$-points mesh based on a $2 \times 2 \times 1$ super-cell is adopted for calculating the dynamical matrix. The thermal expansion coefficient $\alpha$ is investigated based on the Grüneisen approximation, ${ }^{52} \alpha=\frac{1}{N_{k}} \frac{1}{\left.a_{0}{ }^{2} \frac{\partial^{2} E_{\mathrm{s}}}{\partial a^{2}}\right|_{0}} \sum_{j, k} c_{\mathrm{v}}(j, k) \gamma(j, k)$. Here, $N_{k}$ is the $k$-point number adopted in plotting the phonon spectrum, which is equal to 120 in our calculations; $E_{\mathrm{s}}$ is the strain energy; $c_{\mathrm{v}}(j, k)$ is the $(j, k)$ mode contribution to the heat capacity, $c_{\mathrm{v}}(j, k)=k_{\mathrm{B}}\left(\frac{h \omega_{j, k}}{k_{\mathrm{B}} T}\right)^{2} \frac{\exp \left(\frac{h \omega_{j, k}}{k_{\mathrm{B}} T}\right)}{\left[\exp \left(\frac{h \omega_{j, k}}{k_{\mathrm{B}} T}\right)-1\right]^{2}}$. The Specific heat $c$ is proportional to the heat capacity as $c=\frac{1}{N_{k} M} \sum_{j, k} c_{\mathrm{v}}(j, k) .^{53}$

The computational parameters and methods applied in calculating the carrier mobility and thermal properties have been tested in our previous works on $\mathrm{Sc}_{2} \mathrm{CF}_{2}, \mathrm{Sc}_{2} \mathrm{C}(\mathrm{OH})_{2}$ (ref. 54) and $\mathrm{Hf}_{2} \mathrm{CO}_{2}$ (ref. 55) MXenes. The predicted thermal conductivity of graphene in our previous calculation $\left(4.76 \times 10^{3} \mathrm{~W} \mathrm{~m} \mathrm{~m}^{-1} \mathrm{~K}^{-1}\right.$, based on a $5 \mu \mathrm{m}$ flake length at room temperature) is consistent with the experimental results. ${ }^{56}$

\section{Results and discussion}

The geometries and band structure properties of the $\mathrm{F}$ terminated MXenes $\mathrm{Sc}_{3} \mathrm{C}_{2} \mathrm{~F}_{2}, \mathrm{Sc}_{3} \mathrm{~N}_{2} \mathrm{~F}_{2}$ and $\mathrm{Sc}_{3}(\mathrm{CN}) \mathrm{F}_{2}$ are investigated using DFT calculations. As 2D hexagonal materials, the MXenes possess two high-symmetry routes, namely, the $y$ and $x$ directions. ${ }^{57}$ The top view and side view of the $\mathrm{Sc}_{3}(\mathrm{CN}) \mathrm{F}_{2}$ MXene are shown in Fig. 1(a) and (b). The $\mathrm{Sc}_{3} \mathrm{C}_{2} \mathrm{~F}_{2}$ and $\mathrm{Sc}_{3} \mathrm{~N}_{2} \mathrm{~F}_{2}$ have similar structures to $\mathrm{Sc}_{3}(\mathrm{CN}) \mathrm{F}_{2}$ and their side view diagrams are also shown in Fig. 2(a) and (b), respectively. According to our structure models, the $x$-axis coincides with the $x$ direction, and the $y$-axis lies along the $y$ direction. The $\Gamma \rightarrow K(I \rightarrow X)$ and $\Gamma \rightarrow$ $M(\Gamma \rightarrow Y)$ vectors in the Brillouin zone correspond to the realspace $x$ and $y$ directions as shown in Fig. 1(c), respectively. The two carbon or nitrogen layers are sandwiched between three Sc layers, and two fluorine layers are projected onto the central Sc layer. Table 1 lists the lattice constants, formation energies and atomic layer distances marked in Fig. 2. The optimized lattice constant of $\mathrm{Sc}_{3}(\mathrm{CN}) \mathrm{F}_{2}$ is similar to that of $\mathrm{Sc}_{3} \mathrm{C}_{2} \mathrm{~F}_{2}$, and the formation energy is between that of $\mathrm{Sc}_{3} \mathrm{C}_{2} \mathrm{~F}_{2}$ and $\mathrm{Sc}_{3} \mathrm{~N}_{2} \mathrm{~F}_{2}$. As with the result of the substituted $\mathrm{C} / \mathrm{N}$ atoms, the $\mathrm{Sc}-\mathrm{F}$ atomic layer distances are only slightly affected, while the Sc-C and Sc-

Table 1 The lattice constants, formation energies and atomic layer distances obtained by structure relaxation

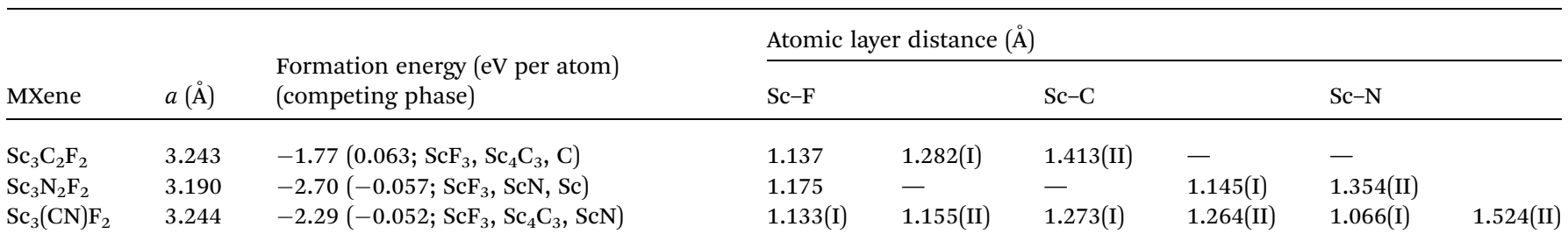


$\mathrm{N}$ distances show notable variations, especially for the bonds with center Sc atoms (labelled as II in Table 1). The band structures of $\mathrm{Sc}_{3} \mathrm{C}_{2} \mathrm{~F}_{2}, \mathrm{Sc}_{3} \mathrm{~N}_{2} \mathrm{~F}_{2}$ and $\mathrm{Sc}_{3}(\mathrm{CN}) \mathrm{F}_{2}$ are also provided in Fig. 2 (vacuum energy is set as zero). Both $\mathrm{Sc}_{3} \mathrm{C}_{2} \mathrm{~F}_{2}$ and $\mathrm{Sc}_{3} \mathrm{~N}_{2} \mathrm{~F}_{2}$ exhibit metallic properties with the Fermi level crossed by energy bands and with band gaps above/below the Fermi levels, while $\mathrm{Sc}_{3}(\mathrm{CN}) \mathrm{F}_{2}$ is determined to be a semiconductor with an indirect band gap of $1.18 \mathrm{eV}$ from HSE06. From the band structure plots, the three $\mathrm{F}$ terminated MXenes also exhibit similar shapes near the Fermi level despite the difference in band gaps. The band gap can also be observed from the partial density of states (PDOS) plot for $\mathrm{Sc}_{3}(\mathrm{CN}) \mathrm{F}_{2}$ in Fig. 3. From the figure, $\mathrm{Sc}$ and $\mathrm{N}$ overlap from -4.5 to $-2.5 \mathrm{eV}$, while $\mathrm{Sc}$ and $\mathrm{C}$ are from -2.5 to $0 \mathrm{eV}$ (forming CBM) near the Fermi level. $\mathrm{Sc}_{3} \mathrm{C}_{2} \mathrm{~F}_{2}$ and $\mathrm{Sc}_{3}(\mathrm{CN}) \mathrm{F}_{2}$ show similar Fermi level energy; since the $\mathrm{Sc}-\mathrm{C}$ bonds are strengthened in $\mathrm{Sc}_{3}(\mathrm{CN}) \mathrm{F}_{2}$ as seen from the reduction of $\mathrm{Sc}-\mathrm{C}$ bond lengths, the $\mathrm{N}$ atoms substitutions lowers the energy of Sc-C hybrid bands forming VBM bands in $\mathrm{Sc}_{3}(\mathrm{CN}) \mathrm{F}_{2}$ relative to that in $\mathrm{Sc}_{3} \mathrm{C}_{2} \mathrm{~F}_{2}$ around the Fermi level. Similarly, the CBM energy of $\mathrm{Sc}_{3}(\mathrm{CN}) \mathrm{F}_{2}$ is raised relative to the corresponding bands in $\mathrm{Sc}_{3} \mathrm{~N}_{2} \mathrm{~F}_{2}$. These result in a rise of the band gap for $\mathrm{Sc}_{3}(\mathrm{CN}) \mathrm{F}_{2}$ at a particular $\mathrm{C} / \mathrm{N}$ ratio. This implies band engineering can be achieved in Sc-based MXenes by the structural design of the MXene, thus expanding the group of semiconducting MXenes.

With the semiconducting MXene $\mathrm{Sc}_{3}(\mathrm{CN}) \mathrm{F}_{2}$ investigated in this work, its carrier mobilities with all the required parameters are then calculated and given in Table 2. From the table, the electron mobility of $\mathrm{Sc}_{3}(\mathrm{CN}) \mathrm{F}_{2}$ by $\mathrm{CBM}$, the red curve in

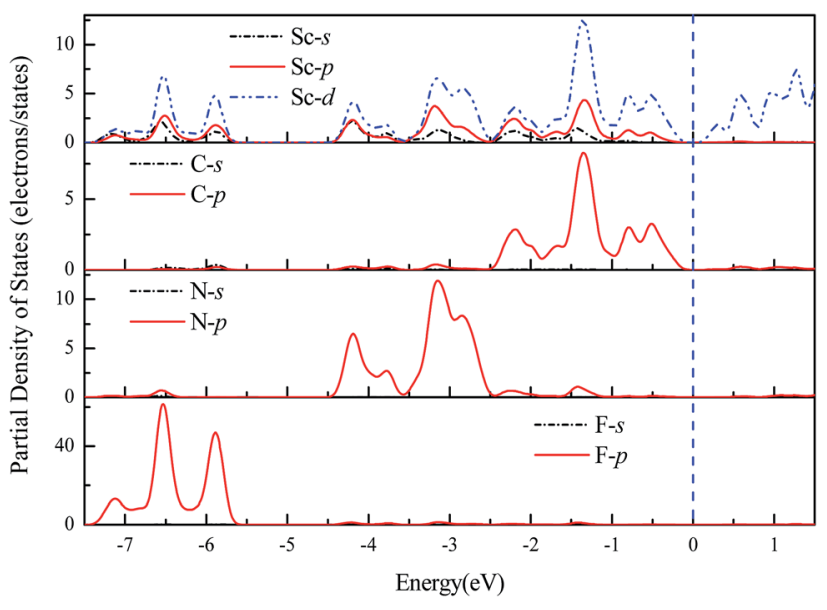

Fig. 3 Partial density of states for the $\mathrm{Sc}_{3}(C N) F_{2}$ MXene.
Fig. 1(d), appears to be highly anisotropic, i.e. $1.348 \times 10^{3} \mathrm{~cm}^{2}$ $\mathrm{V}^{-1} \mathrm{~s}^{-1}$ along the $x(\Gamma \rightarrow X)$ and $0.319 \times 10^{3} \mathrm{~cm}^{2} \mathrm{~V}^{-1} \mathrm{~s}^{-1}$ along the $y(\Gamma \rightarrow Y)$ directions, respectively. For the hole mobilities, two quasi-degenerated sub-bands are present at the VBM as Fig. 1(d) indicates, and we distinguish the two sub-bands as "h1" (orange) and "h2" (blue), respectively. Both of the subbands have been calculated, and the total hole mobilities can be estimated as the statistical average of the two sub-bands on the basis of the Boltzmann distribution. Accordingly, the hole mobilities are determined to be $0.078 \times 10^{3}$ along the $x$ and along the $y$ directions for the "h1" sub-band, and are $0.956 \times$ $10^{3}$ along the $x$ and $1.003 \times 10^{3} \mathrm{~cm}^{2} \mathrm{~V}^{-1} \mathrm{~s}^{-1}$ along the $y$ directions for the "h2" sub-band, respectively. From Table 2, one may note that, for the "h1" or "h2" sub-band, the values of the effective mass and deformation potential constant along the $x$ and $y$ directions are close to each other, analogous to the $\mathrm{Sc}_{2} \mathrm{CT}_{2}$ MXenes calculated in our previous work. ${ }^{54}$ The average hole mobilities of $\mathrm{Sc}_{3}(\mathrm{CN}) \mathrm{F}_{2}$ are $0.517 \times 10^{3}$ along $x$ and $0.540 \times 10^{3}$ $\mathrm{cm}^{2} \mathrm{~V}^{-1} \mathrm{~s}^{-1}$ along the $y$ directions, respectively. Consequently, the predicted hole mobilities for $\mathrm{Sc}_{3}(\mathrm{CN}) \mathrm{F}_{2}$ are almost isotropic. The details of the carrier effective mass calculations are provided in the ESI. $\dagger$ Actually, the electron mobilities are slightly lower than that of $\mathrm{Sc}_{2} \mathrm{CF}_{2}$ and $\mathrm{Sc}_{2} \mathrm{C}(\mathrm{OH})_{2}$, while the hole mobilities are higher than that of $\mathrm{Sc}_{2} \mathrm{CF}_{2}$ and $\mathrm{Sc}_{2} \mathrm{C}(\mathrm{OH})_{2} \cdot{ }^{30}$ The predicted carrier mobilities are much higher than that of monolayer $\mathrm{MoS}_{2},{ }^{58}$ providing a hopeful application in nanoelectronics devices for the $\mathrm{Sc}_{3}(\mathrm{CN}) \mathrm{F}_{2}$ MXene. Moreover, in order to exclude the impact of structural disorder, i.e. the entropy effect on the semiconducting nature of $\mathrm{Sc}_{3}(\mathrm{CN}) \mathrm{F}_{2}$, the possibilities of a random distribution of $\mathrm{C}$ and $\mathrm{N}$ atoms are taken into consideration as well. Three $2 \times 2 \times 1$ super-cells with different $\mathrm{C}$ and $\mathrm{N}$ arrangement models are built for band structure calculations as shown in Fig. 4. Model 0 represents the ordered arrangement of $\mathrm{C}$ and $\mathrm{N}$, and Model 1 and 2 are disordered ones. The results confirm that the $\mathrm{Sc}_{3}(\mathrm{CN}) \mathrm{F}_{2}$ MXene is a semiconductor and imply that the random $\mathrm{C}$ and $\mathrm{N}$ distribution can lead to a slight sub band splitting of CBM and VBM, while the slopes of the bands near the Fermi level keep similar trends, suggesting that the ordered or disordered $\mathrm{Sc}_{3}(\mathrm{CN}) \mathrm{F}_{2}$ MXene might have similar carrier mobilities.

The $\mathrm{Sc}_{3} \mathrm{C}_{2} \mathrm{~F}_{2}, \quad \mathrm{Sc}_{3} \mathrm{~N}_{2} \mathrm{~F}_{2}$ and $\mathrm{Sc}_{3}(\mathrm{CN}) \mathrm{F}_{2}$ MXenes phonon dispersions along $\Gamma \rightarrow M \rightarrow K \rightarrow \Gamma$ are given in Fig. 5 . From the figure, the absence of imaginary phonon frequencies implies the structural stabilities of those MXenes. It is well known that thermal conductivities for semiconductive materials are dominantly contributed by phonon transport. Therefore, the lattice thermal conductivities for $\mathrm{Sc}_{3}(\mathrm{CN}) \mathrm{F}_{2}$ are thus investigated in the

Table 2 The carrier mobilities of $\mathrm{Sc}_{3}(\mathrm{CN}) \mathrm{F}_{2}$. Carrier type "e" and " $\mathrm{h}$ " denote "electron" and "hole", respectively. $m_{x}^{*}$ and $m_{y}^{*}$ are the effective masses along the $x$ and $y$ directions. $E_{x}$ and $E_{y}$ are the deformation potential constants, $C_{x}$ and $C_{y}$ are the elastic moduli. $\mu_{x}$ and $\mu_{y}$ are the roomtemperature carrier mobilities

\begin{tabular}{lccrrrrrr}
\hline Carrier type & $m_{x}^{*} / m_{y}^{*}$ & $m_{y}^{*} / m_{0}$ & $E_{x}(\mathrm{eV})$ & $E_{y}(\mathrm{eV})$ & $C_{x}\left(\mathrm{~J} \mathrm{~m}^{-2}\right)$ & $C_{y}\left(\mathrm{~J} \mathrm{~m}^{-2}\right)$ & $\mu_{x}\left(10^{3} \mathrm{~cm}^{2} \mathrm{~V}^{-1} \mathrm{~s}^{-1}\right)$ & $\mu_{y}\left(10^{3} \mathrm{~cm}^{2} \mathrm{~V}^{-1} \mathrm{~s}^{-1}\right)$ \\
\hline $\mathrm{e}$ & 0.21 & 1.70 & 5.722 & 4.144 & 262.15 & 262.17 & 1.348 & 0.319 \\
$\mathrm{~h} 1$ & 2.93 & 2.81 & 4.422 & 4.622 & 262.15 & 262.17 & 0.078 & 0.078 \\
$\mathrm{~h} 2$ & 0.51 & 0.53 & -3.098 & -2.894 & 262.15 & 262.17 & 0.956 & 1.003
\end{tabular}




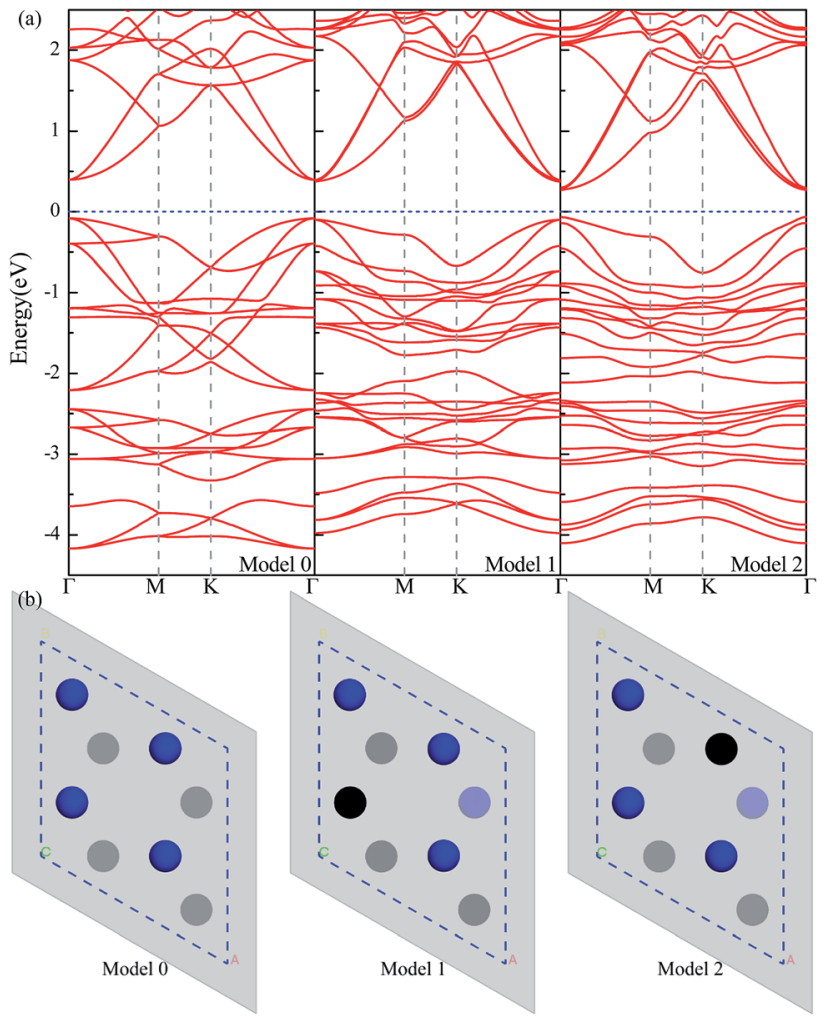

Fig. 4 (a) Band structures of $\mathrm{Sc}_{3}(\mathrm{CN}) \mathrm{F}_{2}$ in Model 0, 1 and 2, the Fermi level located at zero; (b) the $\mathrm{C}$ (black) and $\mathrm{N}$ (blue) atoms arrangement models sketch, atoms under the gray semi-transparent interfaces present the second layer atoms.

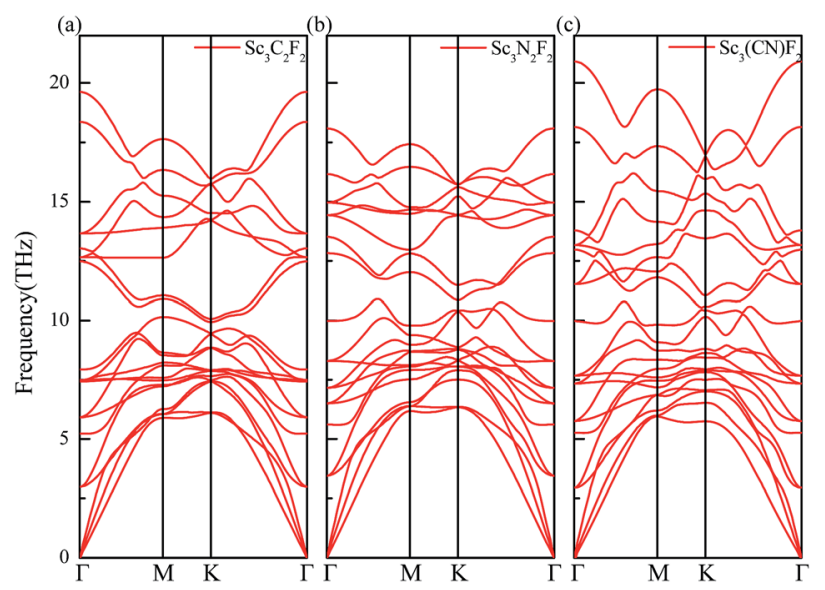

Fig. 5 The phonon dispersions of the $\mathrm{SC}_{3} \mathrm{C}_{2} \mathrm{~F}_{2}$ (a), $\mathrm{SC}_{3} \mathrm{~N}_{2} \mathrm{~F}_{2}$ (b) and $\mathrm{Sc}_{3}(\mathrm{CN}) \mathrm{F}_{2}$ (c) MXenes.

current work and the electronic thermal conductivity for $\mathrm{Sc}_{3}(\mathrm{CN}) \mathrm{F}_{2}$ is considered negligible. The values for the $\mathrm{Sc}_{3}(\mathrm{CN}) \mathrm{F}_{2}$ MXene are calculated according to eqn (2) based on the phonon dispersions. The required parameters, including the group velocity $v_{j}$, Grüneisen parameter $\gamma_{j}$ and the square of the Grüneisen parameter $\left\langle\gamma_{j}^{2}\right\rangle$ are list in Table 3. From the table, the group velocities along the $\Gamma \rightarrow M$ (real-space $y$ ) direction for
Table 3 The group velocity $v_{j}$, the Grüneisen parameter $\gamma_{j}$ and the square of the Grüneisen parameter $\left\langle\gamma_{j}^{2}\right\rangle$ for calculating the thermal conductivities of $\mathrm{Sc}_{3}(\mathrm{CN}) \mathrm{F}_{2}$

\begin{tabular}{|c|c|c|c|c|c|c|c|c|c|}
\hline & \multicolumn{3}{|c|}{$v_{j}\left(\mathrm{~m} \mathrm{~s}^{-1}\right)$} & \multicolumn{3}{|l|}{$\underline{\gamma_{j}}$} & \multicolumn{3}{|l|}{$\left\langle\gamma_{j}^{2}\right\rangle$} \\
\hline & TA & LA & $\mathrm{ZA}$ & TA & LA & $\mathrm{ZA}$ & TA & LA & $\mathrm{ZA}$ \\
\hline$\Gamma \rightarrow M$ & 3387 & 3897 & 3301 & 2.901 & 1.189 & 0.942 & 8.685 & 2.254 & 0.899 \\
\hline$\Gamma \rightarrow K$ & 3220 & 3431 & 2758 & 1.909 & 1.574 & 1.893 & 4.263 & 2.591 & 4.032 \\
\hline
\end{tabular}

transversal acoustic (TA), longitudinal acoustic (LA) and out-ofplane acoustic (ZA) modes are larger than the $I \rightarrow K$ (real-space $x$ ) direction. In particular, the group velocity values for the ZA mode along $\Gamma \rightarrow M$ are approximately $20 \%$ higher. Moreover, the minimum values for Grüneisen parameter $\gamma_{j}$ and $\left\langle\gamma_{j}^{2}\right\rangle$ found originated from the ZA mode along the $\Gamma \rightarrow M$ direction. For the $\Gamma \rightarrow K$ direction, the minimum in $\gamma_{j}$ and $\left\langle\gamma_{j}^{2}\right\rangle$ occurs in the LA mode. The ratio of $\left\langle\gamma_{j}^{2}\right\rangle$ between the $\Gamma \rightarrow K$ and $I \rightarrow M$ directions is the maximum by the ZA mode. These may imply that the out of plane phonon modes are responsible for anisotropy in thermal conductance. Similar phenomena can be found in the parameters for calculating the thermal conductivities of $\mathrm{Sc}_{2} \mathrm{CF}_{2}, \mathrm{Zr}_{2} \mathrm{CO}_{2}$ and $\mathrm{Hf}_{2} \mathrm{CO}_{2}$ MXenes. Based on the parameters obtained, the thermal conductivities of $\mathrm{Sc}_{3}(\mathrm{CN}) \mathrm{F}_{2}$ have been calculated.

The thermal conductivity is dependent upon the flake length $d$ due to the existence of boundary scattering. The theoretical temperature dependence thermal conductivity of $\mathrm{Sc}_{3}(\mathrm{CN}) \mathrm{F}_{2}$ with flake lengths of $5 \mu \mathrm{m}$ along the $\Gamma \rightarrow M$ and $\Gamma \rightarrow K$ directions with TA, LA and ZA contributions are plotted in Fig. 6(a) and (b), respectively. The ZA mode has the highest contribution to the theoretical thermal conductivity along the $\Gamma$ $\rightarrow M$ direction, due to the small value for the square of the Grüneisen parameter $\left\langle\gamma_{j}^{2}\right\rangle$, and the same is for the LA mode along the $\Gamma \rightarrow M$ direction. At room temperature (300 K), the calculated total thermal conductivities with TA, LA and ZA contributions along the $\Gamma \rightarrow M$ and $\Gamma \rightarrow K$ directions are 179 and $75.0 \mathrm{~W} \mathrm{~m}^{-1} \mathrm{~K}^{-1}$, respectively. The anisotropy in thermal conductivity is similar with that for other MXenes such as $\mathrm{Sc}_{2} \mathrm{CF}_{2}, \mathrm{Sc}_{2} \mathrm{C}(\mathrm{OH})_{2},{ }^{54} \mathrm{Ti}_{2} \mathrm{CO}_{2}, \mathrm{Zr}_{2} \mathrm{CO}_{2}$ and $\mathrm{Hf}_{2} \mathrm{CO}_{2},{ }^{55}$ demonstrating that anisotropic thermal conductivity may be a common feature for semiconducting MXenes including $\mathrm{Sc}_{3}(\mathrm{CN}) \mathrm{F}_{2}$. The temperature dependent thermal conductivities for the $\mathrm{Sc}_{3}(\mathrm{CN}) \mathrm{F}_{2}$ MXene with flake lengths of 1-100 $\mu \mathrm{m}$ along the $I \rightarrow M$ and $I \rightarrow K$ directions are shown in Fig. 6(c) and (d), respectively. From the figure, the thermal conductivity increases monotonically with increasing flake length in both directions, and is more sensitive to the flake length at low temperatures. The room temperature thermal conductivity along the $\Gamma \rightarrow M$ direction increases from 123 to $283 \mathrm{~W} \mathrm{~m}^{-1} \mathrm{~K}^{-1}$ as the flake length increases from 1 to $100 \mu \mathrm{m}$, which can be understood as analogous to grain size controlled thermal conductivity for bulk materials. Comparatively, the thermal conductivity along the $\Gamma$ $\rightarrow K$ direction increases from 55.7 to $111 \mathrm{~W} \mathrm{~m}^{-1} \mathrm{~K}^{-1}$, approximately half of that in the $\Gamma \rightarrow M$ direction. Despite that the 

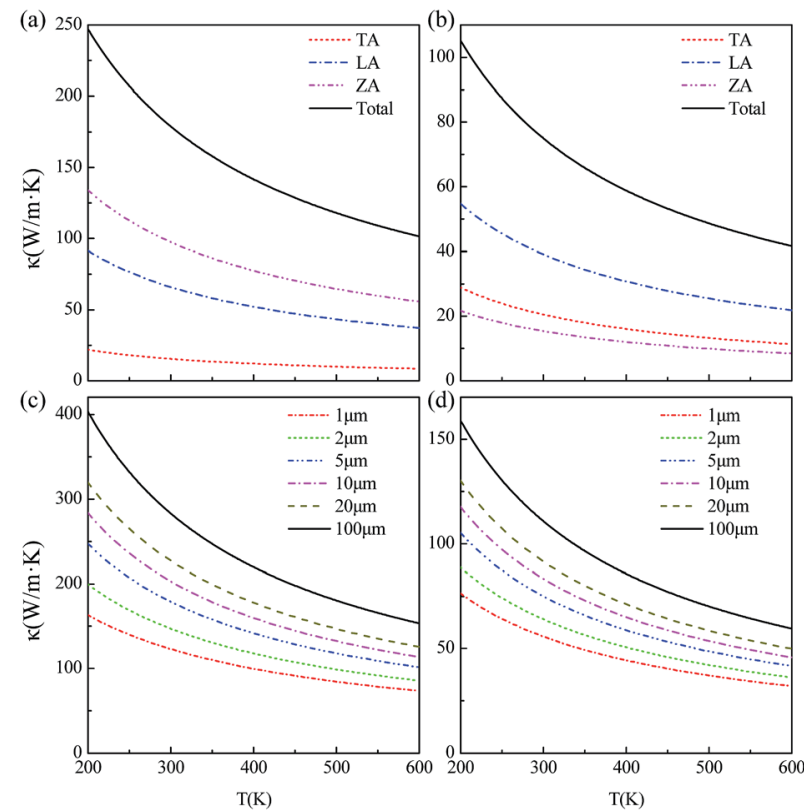

Fig. 6 The temperature dependence thermal conductivities for the $\mathrm{Sc}_{3}(\mathrm{CN}) \mathrm{F}_{2}$ MXene along the $\Gamma \rightarrow M(\mathrm{a})$ and $I \rightarrow K(\mathrm{~b})$ directions with 5 $\mu \mathrm{m}$ flake length with TA, LA and ZA contributions. The temperature dependence thermal conductivities for the $\mathrm{SC}_{3}(\mathrm{CN}) \mathrm{F}_{2}$ MXene with 1$100 \mu \mathrm{m}$ flake lengths along the $\Gamma \rightarrow M(\mathrm{c})$ and $\Gamma \rightarrow K(\mathrm{~d})$ directions.

(a)

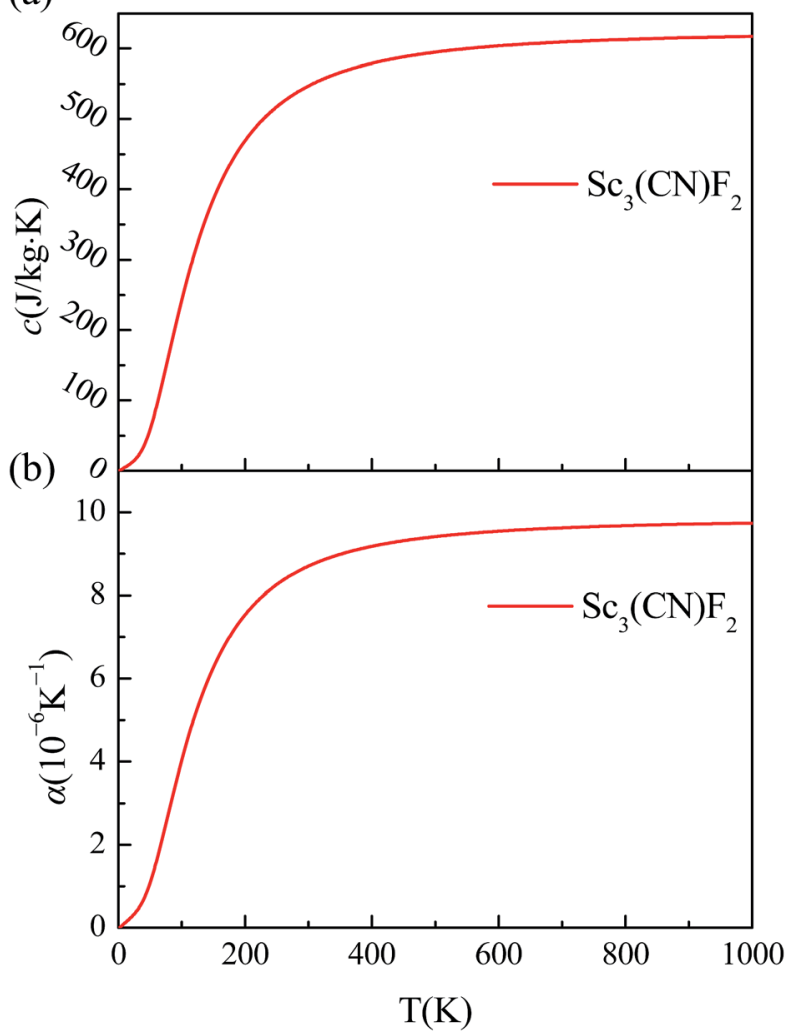

Fig. 7 (a) The temperature dependence of $\mathrm{Sc}_{3}(\mathrm{CN}) \mathrm{F}_{2}$ specific heat. (b) The temperature dependence of the $\mathrm{Sc}_{3}(\mathrm{CN}) \mathrm{F}_{2}$ thermal expansion coefficient. room temperature thermal conductivity is much lower than the values in the range $4.84( \pm 0.44) \times 10^{3}$ to $5.30( \pm 0.48) \times 10^{3} \mathrm{~W}$ $\mathrm{m}^{-1} \mathrm{~K}^{-1}$ for single-layer graphene, ${ }^{56}$ the values for $\mathrm{Sc}_{3}(\mathrm{CN}) \mathrm{F}_{2}$ and other MXenes like $\mathrm{Sc}_{2} \mathrm{CT}_{2}(\mathrm{~T}=\mathrm{F}, \mathrm{OH})$ are of the same order of magnitude, higher than that of the phosphorene ${ }^{59}$ and monolayer $\mathrm{MoS}_{2}{ }^{60}$ These results indicate that the $\mathrm{Sc}_{3}(\mathrm{CN}) \mathrm{F}_{2}$ possesses good heat dissipation performance if used as an electronic device.

The specific heat and thermal expansion coefficient are also studied from the phonon dispersion for the hexagonal $\mathrm{BZ}$ of $\mathrm{Sc}_{3}(\mathrm{CN}) \mathrm{F}_{2}$, and the corresponding temperature dependence for $\mathrm{Sc}_{3}(\mathrm{CN}) \mathrm{F}_{2}$ are shown in Fig. 7 (a) and (b). These results suggest that both the specific heat and thermal expansion coefficient are positively related to the temperature, and the room temperature values are $547 \mathrm{~J} \mathrm{~kg}^{-1} \mathrm{~K}^{-1}$ and $8.703 \times 10^{-6} \mathrm{~K}^{-1}$, respectively. By contrast, the specific heat and thermal expansion coefficient are $385 \mathrm{~J} \mathrm{~kg}^{-1} \mathrm{~K}^{-1}$ and $16.5 \times 10^{-6} \mathrm{~K}^{-1}$ for copper, and $412 \mathrm{~J} \mathrm{~kg}^{-1} \mathrm{~K}^{-1}$ and $11.8 \times 10^{-6} \mathrm{~K}^{-1}$ for iron. In addition, the room temperature specific heat is much higher than the value of $238 \mathrm{~J} \mathrm{~kg}^{-1} \mathrm{~K}^{-1}$ due to the relatively small relative atomic mass of Sc and the thermal expansion coefficient is close to the value of $6.094 \times 10^{-6} \mathrm{~K}^{-1}$ for $\mathrm{Hf}_{2} \mathrm{CO}_{2}$ MXene. ${ }^{55}$ The relatively high specific heat and low thermal expansion coefficient make $\mathrm{Sc}_{3}(\mathrm{CN}) \mathrm{F}_{2}$ a good candidate material for nanoelectronic devices.

\section{Conclusions}

In this work, we report our design and theoretical calculations of the semiconducting MXene $\mathrm{Sc}_{3}(\mathrm{CN}) \mathrm{F}_{2}$. Different from the mother metallic $\mathrm{Sc}_{3} \mathrm{C}_{2} \mathrm{~F}_{2}$ and $\mathrm{Sc}_{3} \mathrm{~N}_{2} \mathrm{~F}_{2}$ MXenes, the $\mathrm{Sc}_{3}(\mathrm{CN}) \mathrm{F}_{2}$ MXene is a semiconductor with an indirect band gap of $1.18 \mathrm{eV}$ from the HSE06 band structures analysis. The electrical and thermal properties of the $\mathrm{Sc}_{3}(\mathrm{CN}) \mathrm{F}_{2}$ MXene are subsequently predicted by the current computational study. The $\mathrm{Sc}_{3}(\mathrm{CN}) \mathrm{F}_{2}$ presents great anisotropy in electron mobility, and approximate isotropy in hole mobility. The electron mobilities of $\mathrm{Sc}_{3}(\mathrm{CN}) \mathrm{F}_{2}$ are $1.348 \times 10^{3}$ along $x$ and $0.319 \times$ $10^{3} \mathrm{~cm}^{2} \mathrm{~V}^{-1} \mathrm{~s}^{-1}$ along the $y$ directions, and the hole mobilities are $0.517 \times 10^{3}$ along $x$ and $0.540 \times 10^{3} \mathrm{~cm}^{2} \mathrm{~V}^{-1} \mathrm{~s}^{-1}$ along the $y$ directions, respectively. The thermal conductivities for the $\mathrm{Sc}_{3}(\mathrm{CN}) \mathrm{F}_{2}$ are studied with flake lengths of 1-100 $\mu \mathrm{m}$. The thermal conductivity increases monotonically with increasing flake length, and the room temperature thermal conductivity along the $\Gamma \rightarrow M$ direction is $179 \mathrm{~W} \mathrm{~m}^{-1} \mathrm{~K}^{-1}$ with a flake length of $5 \mu \mathrm{m}$. In addition, the relatively high specific heat and low thermal expansion coefficient make $\mathrm{Sc}_{3}(\mathrm{CN}) \mathrm{F}_{2}$ a good candidate material for nanoelectronic devices. The computational data provided here is expected to be meaningful for the expansion of the MXene family towards applications in electronic devices.

\section{Conflicts of interest}

There are no conflicts to declare. 


\section{Acknowledgements}

The present work was supported by the National Key Research and Development Program of China (No. 2016YFB0700100), the Ningbo Municipal Key Project (2014S10001), the National Natural Science Foundation of China (Grant No. 91226202 and 91426304), the "Strategic Priority Research Program" of the Chinese Academy of Sciences (Grant No. XDA02040105 and XDA03010305), the Key Research Program of Frontier Sciences, CAS (Grant No. QYZDB-SSW-JSC037), One Thousand Youth Talents plan, ITaP at Purdue University and the Special Program for Applied Research on Super Computation of the NSFCGuangdong Joint Fund (the second phase) (U1501501 to Juan $\mathrm{Li}$ and Aiguo $\mathrm{Wu}$ ) for computing resources, the key technology of nuclear energy 2014 CAS Interdisciplinary Innovation Team, and the Major Project of the Ministry of Science and Technology of China (Grant No. 2015ZX06004-001).

\section{Notes and references}

1 M. Naguib, V. N. Mochalin, M. W. Barsoum and Y. Gogotsi, Adv. Mater., 2014, 26, 982.

2 Q. Hu, D. Sun, Q. Wu, H. Wang, L. Wang, B. Liu, A. Zhou and J. He, J. Phys. Chem. A, 2013, 117, 14253-14260.

3 V. M. Hong Ng, H. Huang, K. Zhou, P. S. Lee, W. Que, J. Z. Xu and L. B. Kong, J. Mater. Chem. A, 2017, 5, 3039-3068.

4 M. Khazaei, A. Ranjbar, M. Arai, T. Sasaki and S. Yunoki, J. Mater. Chem. C, 2017, 5, 2488-2503.

5 B. Anasori, M. R. Lukatskaya and Y. Gogotsi, Nat. Rev. Mater., 2017, 2, 16098.

6 P. Eklund, M. Beckers, U. Jansson, H. Högberg and L. Hultman, Thin Solid Films, 2010, 518, 1851-1878.

7 P. Eklund, J. Rosen and P. O. Å. Persson, J. Phys. D: Appl. Phys., 2017, 50, 113001.

8 B. Anasori, M. Dahlqvist, J. Halim, E. J. Moon, J. Lu, B. C. Hosler, E. N. Caspi, S. J. May, L. Hultman, P. Eklund, J. Rosén and M. W. Barsoum, J. Appl. Phys., 2015, 118, 94304.

9 J. Lei, X. Zhang and Z. Zhou, Front. Physiol., 2015, 10, 276286.

10 M. Naguib, M. Kurtoglu, V. Presser, J. Lu, J. Niu, M. Heon, L. Hultman, Y. Gogotsi and M. W. Barsoum, Adv. Mater., 2011, 23, 4248-4253.

11 M. Naguib, O. Mashtalir, J. Carle, V. Presser, J. Lu, L. Hultman, Y. Gogotsi and M. W. Barsoum, ACS Nano, 2012, 6, 1322-1331.

12 U. Yorulmaz, A. Özden, N. K. Perkgöz, F. Ay and C. Sevik, Nanotechnology, 2016, 27, 335702.

13 F. Shahzad, M. Alhabeb, C. B. Hatter, B. Anasori, S. Man Hong, C. M. Koo and Y. Gogotsi, Science, 2016, 353, 11371140.

14 B. Anasori, Y. Xie, M. Beidaghi, J. Lu, B. C. Hosler, L. Hultman, P. R. C. Kent, Y. Gogotsi and M. W. Barsoum, ACS Nano, 2015, 9, 9507-9516.

15 C. Si, J. You, W. Shi, J. Zhou and Z. Sun, J. Mater. Chem. C, 2016, 4, 11524-11529.

16 C. Xu, L. Wang, Z. Liu, L. Chen, J. Guo, N. Kang, X. Ma, H. Cheng and W. Ren, Nat. Mater., 2015, 14, 1135-1141.
17 L. M. Azofra, N. Li, D. R. MacFarlane and C. Sun, Energy Environ. Sci., 2016, 9, 2545-2549.

18 A. Morales-Garcia, A. Fernandez-Fernandez, F. Vines and F. Illas, J. Mater. Chem. A, 2018, 6, 3381-3385.

19 H. Weng, A. Ranjbar, Y. Liang, Z. Song, M. Khazaei, S. Yunoki, M. Arai, Y. Kawazoe, Z. Fang and X. Dai, Phys. Rev. B: Condens. Matter Mater. Phys., 2015, 92, 75436.

20 M. Khazaei, A. Ranjbar, M. Arai and S. Yunoki, Phys. Rev. B, 2016, 94, 125152.

21 H. Fashandi, V. Ivády, P. Eklund, A. L. Spetz, M. I. Katsnelson and I. A. Abrikosov, Phys. Rev. B: Condens. Matter Mater. Phys., 2015, 92, 155142.

22 M. Ashton, K. Mathew, R. G. Hennig and S. B. Sinnott, J. Phys. Chem. C, 2016, 120, 3550-3556.

23 Q. Tang, Z. Zhou and P. Shen, J. Am. Chem. Soc., 2012, 134, 16909-16916.

24 M. Khazaei, M. Arai, T. Sasaki, C. Chung, N. S. Venkataramanan, M. Estili, Y. Sakka and Y. Kawazoe, Adv. Funct. Mater., 2013, 23, 2185-2192.

25 X. Zha, K. Luo, Q. Li, Q. Huang, J. He, X. Wen and S. Du, Europhys. Lett., 2015, 111, 26007.

26 M. Khazaei, M. Arai, T. Sasaki, M. Estili and Y. Sakka, Phys. Chem. Chem. Phys., 2014, 16, 7841-7849.

27 M. Khazaei, A. Ranjbar, M. Ghorbani-Asl, M. Arai, T. Sasaki, Y. Liang and S. Yunoki, Phys. Rev. B, 2016, 93, 205125.

28 B. Anasori, C. Shi, E. J. Moon, Y. Xie, C. A. Voigt, P. R. C. Kent, S. J. May, S. J. L. Billinge, M. W. Barsoum and Y. Gogotsi, Nanoscale Horiz., 2016, 1, 227-234.

29 L. Kaikai, J. Tifeng, X. Ruirui, Z. Guodong, Z. Jingxin, Z. Lexin and P. Qiuming, Sci. China Mater., 2018, 61, 728-736.

30 K. Xiong, P. Wang, G. Yang, Z. Liu, H. Zhang, S. Jin and X. Xu, Sci. Rep., 2017, 7, 15095.

31 S. Kumar and U. Schwingenschl Ogl, Phys. Rev. B, 2016, 94, 35405.

32 J. Liu, X. Kan, B. Amin, L. Gan and Y. Zhao, Phys. Chem. Chem. Phys., 2017, 19, 32253-32261.

33 S. Wang, Y. Du and W. Liao, Chin. Phys. B, 2017, 26, 17806.

34 Y. Lee, Y. Hwang and Y. Chung, ACS Appl. Mater. Interfaces, 2015, 7, 7163-7169.

35 E. Balcı, Ü. Ö. Akkuș and S. Berber, J. Mater. Chem. C, 2017, 5, 5956-5961.

36 G. Kresse and D. Joubert, Phys. Rev. B: Condens. Matter Mater. Phys., 1999, 59, 1758-1775.

37 J. P. Perdew, J. A. Chevary, S. H. Vosko, K. A. Jackson, M. R. Pederson, D. J. Singh and C. Fiolhais, Phys. Rev. B: Condens. Matter Mater. Phys., 1992, 46, 6671-6687.

38 J. Hafner, J. Comput. Chem., 2008, 29, 2044-2078.

39 M. Marsman, J. Paier, A. Stroppa and G. Kresse, J. Phys.: Condens. Matter, 2008, 20, 64201.

40 Y. Wang, S. Wang, Y. Lu, J. Jiang and S. A. Yang, Nano Lett., 2016, 16, 4576-4582.

41 J. Xi, M. Long, L. Tang, D. Wang and Z. Shuai, Nanoscale, 2012, 4, 4348-4369.

42 M. Zhou, X. Chen, M. Li and A. Du, J. Mater. Chem. C, 2017, 5, 1247-1254.

43 J. Bardeen and W. Shockley, Phys. Rev., 1950, 80, 72-80. 44 S. Bruzzone and G. Fiori, Appl. Phys. Lett., 2011, 99, 222108. 
45 R. Fei and L. Yang, Nano Lett., 2014, 14, 2884-2889.

46 P. G. Klemens and D. F. Pedraza, Carbon, 1994, 32, 735-741.

47 P. G. Klemens, Int. J. Thermophys., 2001, 22, 265-275.

48 T. Li, Phys. Rev. B: Condens. Matter Mater. Phys., 2012, 85, 235407.

49 S. Grimme, J. Antony, S. Ehrlich and H. Krieg, J. Chem. Phys., 2010, 132, 154104.

50 A. Togo, F. Oba and I. Tanaka, Phys. Rev. B: Condens. Matter Mater. Phys., 2008, 78, 134106.

51 X. Gonze and C. Lee, Phys. Rev. B: Condens. Matter Mater. Phys., 1997, 55, 10355-10368.

52 N. Mounet and N. Marzari, Phys. Rev. B: Condens. Matter Mater. Phys., 2005, 71, 205214.

53 M. Upadhyay Kahaly and U. V. Waghmare, Appl. Phys. Lett., 2007, 91, 23112.
54 X. Zha, J. Zhou, Y. Zhou, Q. Huang, J. He, J. S. Francisco, K. Luo and S. Du, Nanoscale, 2016, 8, 6110-6117.

55 X. Zha, Q. Huang, J. He, H. He, J. Zhai, J. S. Francisco and S. Du, Sci. Rep., 2016, 6, 27971.

56 A. A. Balandin, S. Ghosh, W. Bao, I. Calizo, D. Teweldebrhan, F. Miao and C. N. Lau, Nano Lett., 2008, 8, 902-907.

57 M. Xu, T. Liang, M. Shi and H. Chen, Chem. Rev., 2013, 113, 3766-3798.

58 Y. Cai, G. Zhang and Y. Zhang, J. Am. Chem. Soc., 2014, 136, 6269-6275.

59 R. Fei, A. Faghaninia, R. Soklaski, J. Yan, C. Lo and L. Yang, Nano Lett., 2014, 14, 6393-6399.

60 R. Yan, J. R. Simpson, S. Bertolazzi, J. Brivio, M. Watson, X. Wu, A. Kis, T. Luo, A. R. Hight Walker and H. G. Xing, ACS Nano, 2014, 8, 986-993. 